\title{
The Transit Light Curve Project. XI. Submillimagnitude Photometry of Two Transits of the Bloated Planet WASP- $4 \mathrm{~b}^{1}$
}

\author{
Joshua N. Winn², Matthew J. Holman ${ }^{3}$, Joshua A. Carter², \\ Guillermo Torres ${ }^{3}$, David J. Osip ${ }^{4}$, Thomas Beatty ${ }^{2}$
}

\begin{abstract}
We present photometry of two transits of the giant planet WASP-4b with a photometric precision of 400-800 parts per million and a time sampling of 25-40 s. The two midtransit times are determined to within 6 s. Together with previously published times, the data are consistent with a constant orbital period, giving no compelling evidence for period variations that would be produced by a satellite or additional planets. Analysis of the new photometry, in combination with stellar-evolutionary modeling, gives a planetary mass and radius of $1.237 \pm$ $0.064 M_{\text {Jup }}$ and $1.365 \pm 0.021 R_{\text {Jup }}$. The planet is $15 \%$ larger than expected based on previously published models of solar-composition giant planets. With data of the quality presented here, the detection of transits of a "super-Earth" of radius $1.75 R_{\oplus}$ would have been possible.
\end{abstract}

Subject headings: planetary systems — stars: individual (WASP-4=USNO-B1.0 04790948995)

\section{Introduction}

Wilson et al. (2008) recently reported the discovery of WASP-4b, a giant planet that orbits and transits a G7V star with a period of 1.34 days. This discovery is notable because the planet has an unusually large radius and short orbital period, and the star is one of the

\footnotetext{
${ }^{1}$ Based on observations with the 6.5m Magellan Telescopes located at Las Campanas Observatory, Chile.

${ }^{2}$ Department of Physics, and Kavli Institute for Astrophysics and Space Research, Massachusetts Institute of Technology, Cambridge, MA 02139, USA

${ }^{3}$ Harvard-Smithsonian Center for Astrophysics, 60 Garden Street, Cambridge, MA 02138, USA

${ }^{4}$ Las Campanas Observatory, Carnegie Observatories, Casilla 601, La Serena, Chile
} 
brightest transit hosts that is known in the Southern sky $(V=12.5)$. The large radius seems to place the planet among the "bloated" planets for which there is no clear explanation (see, e.g., Burrows et al. 2007, Guillot 2008). The short period raises the possibility of observing tidal decay (Rasio et al. 1996, Sasselov 2003) and makes WASP-4b an attractive target for observations of occultations (secondary eclipses) that would lead to detections of the reflected light and thermal emission from the planet's atmosphere.

The host star's brightness and southern declination are important for a practical reason: they allow the large-aperture telescopes of the southern hemisphere to be used advantageously. In this paper, we report on observations of a transit of WASP-4b with the Magellan/Baade $6.5 \mathrm{~m}$ telescope, with the goal of deriving independent and refined parameters for this interesting system. Previous papers in this series, the Transit Light Curve project, have achieved this goal by combining the information from many independent transit observations with smaller telescopes (see, e.g., Holman et al. 2006, Winn et al. 2007). In principle, with a larger telescope, it should be possible to achieve this goal with fewer observations and also to measure precise midtransit times, which can be used to search for additional planets via the method of Holman \& Murray (2005) and Agol et al. (2005). Gillon et al. (2008) recently presented photometry of WASP-4 with one of the $8.2 \mathrm{~m}$ Very Large Telescopes, with the same motivation.

This paper is organized as follows. $\S 2$ describes the observations and data reduction, $\S 3$ describes the photometric analysis, $\S 4$ describes the results of stellar-evolutionary modeling, and $\S 5$ discusses the newly-measured midtransit times and a refined ephemeris. Finally, $\S 6$ discusses the refined measurement of the planetary radius, and considers how small a planet we could have detected, given data of the quality presented here.

\section{Observations and Data Reduction}

We observed the transits of UT 2008 Aug 19 and 2008 Oct 09 with the Baade 6.5m telescope, one of the two Magellan telescopes at Las Campanas Observatory in Chile. We used the Raymond and Beverly Sackler Magellan Instant Camera (MagIC) and its SITe $2048 \times 2048$ pixel CCD detector, with a scale of 0 '.069 pixel $^{-1}$. Ordinarily this detector uses four amplifiers, each of which reads a quadrant of the array, giving a total readout time of 23 s. We used a $2048 \times 256$ pixel subarray and a single amplifier, giving a readout time of $10 \mathrm{~s}$. We rotated the field of view to align WASP-4 and a nearby comparison star along the

long axis of the subarray (parallel to the read register). The comparison star is $36^{\prime \prime}$ east and $71^{\prime \prime}$ south of WASP-4. At the start of each night, we verified that the time stamps recorded by MagIC were in agreement with GPS-based times to within one second. On each night 
we obtained repeated $z$-band exposures of WASP-4 and the comparison star for about $5 \mathrm{hr}$ bracketing the predicted transit time. Autoguiding kept the image registration constant to within 10 pixels over the course of the night.

During the 2008 Aug 19 observations, WASP-4 rose from an airmass of 1.09 to 1.03 , and then set to an airmass of 1.23. At first, we used an exposure time of $30 \mathrm{~s}$. Shortly after midtransit the seeing improved abruptly, from a full-width at half-maximum (FWHM) of 11 pixels to 7 pixels. As a result of the higher rate of detected photons per pixel, some images were spoiled due to nonlinearity and saturation. For the rest of the night we used an exposure time of $t_{\exp }=15 \mathrm{~s}$.

During the 2008 Oct 09 observations, WASP-4 rose from an airmass of 1.10 to 1.03 , and then set to an airmass of 1.21. Having learned our lesson in August, we deliberately defocused the telescope so that the image width was dominated by the effects of the telescope aberration rather than natural seeing. This was done by moving the secondary mirror by a constant displacement relative to the in-focus position determined by the guider probe. The exposure time was $t_{\exp }=30 \mathrm{~s}$. The stellar images were "donuts" with a diameter of approximately 25 pixels.

We used standard IRAF procedures for overscan correction, trimming, bias subtraction, and flat-field division. The bias frame was calculated from the median of 60 zero-second exposures, and the flat field for each night was calculated from the median of $60 z$-band exposures of a dome flat screen. We performed aperture photometry of WASP-4 and the comparison star and divided the flux of WASP-4 by the flux of the comparison star. We experimented with different aperture sizes and sky regions, aiming to minimize the variations in the out-of-transit (OOT) portion of the differential light curve. Best results were obtained with an aperture radius of 38 pixels for the 2008 Aug 19 observations and 35 pixels for the 2008 Oct 09 observations.

A few images were also obtained with Johnson-Cousins $V R I$ filters to measure the difference in color between WASP-4 and the comparison star. The instrumental magnitude differences (target minus WASP-4) were $\Delta V=0.434, \Delta R=0.469, \Delta I=0.478$, and $\Delta z=$ 0.472. Evidently the two stars are similar in color, with the comparison star being slightly bluer $[\Delta(V-I)=-0.044]$. As described in $\S 3$, the $z$-band time series was corrected for differential extinction between the target and comparison star by fitting a linear function of airmass to the magnitude difference. The results were consistent with the expectation that the bluer comparison star suffers from greater extinction per unit airmass. The extinctioncorrected data are given in Table 1, and plotted in Fig. 1, along with the best-fitting model.

Due to the abrupt seeing change on 2008 Aug 19 and the associated change in exposure 

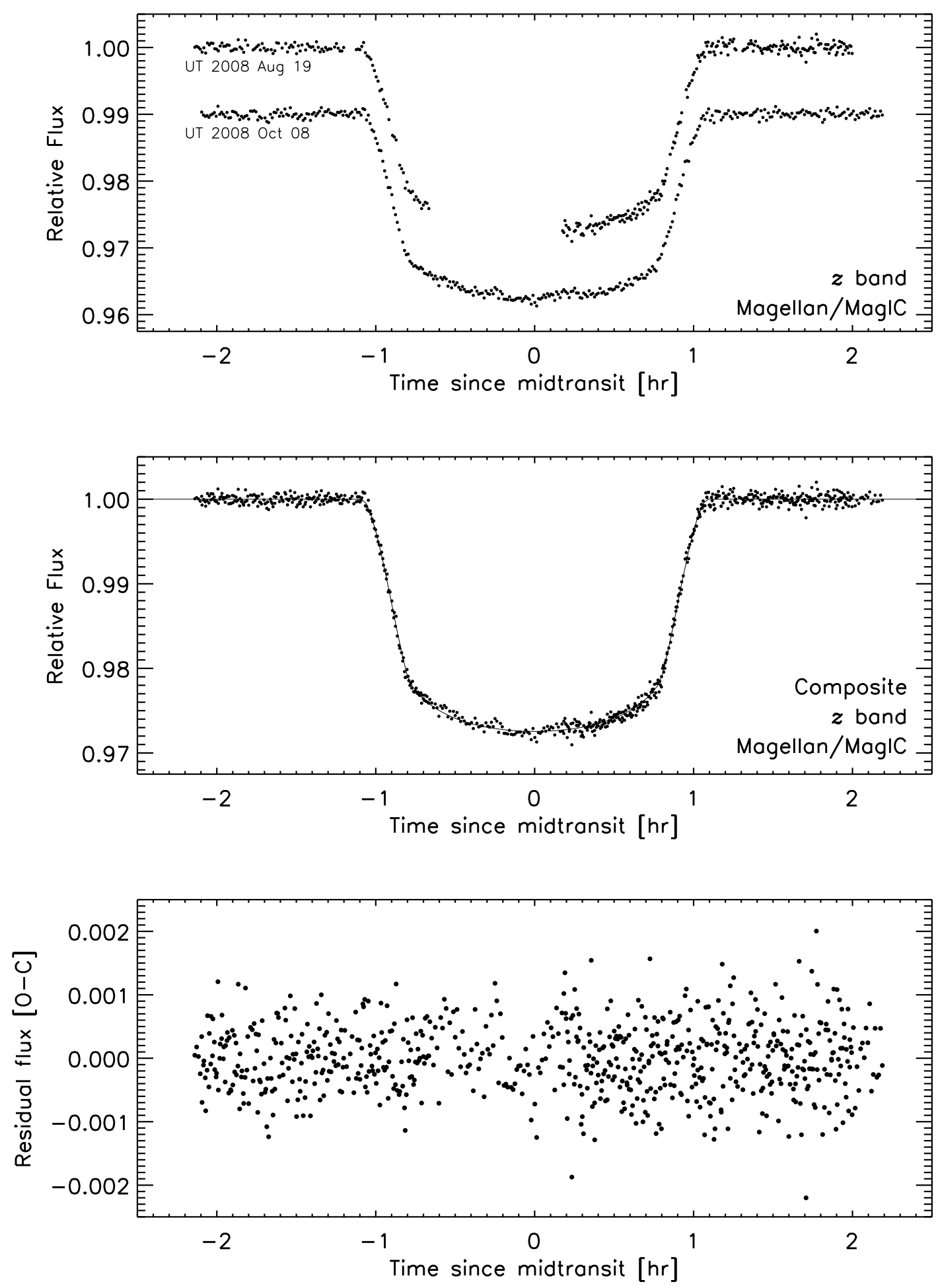

Fig. 1.- Top.-Relative $z$-band photometry of WASP-4 based on observations with the Magellan (Baade) 6.5m telescope. Middle.-Composite light curve. The solid line shows the best-fitting model. Bottom.-Residuals between the data and the best-fitting model. 
level, we consider the $15 \mathrm{~s}$ and $30 \mathrm{~s}$ exposures as two separate time series (TS1 and TS2). Thus, together with the 2008 Oct 09 time series (TS3), there were 3 time series to be analyzed. The TS1 pre-ingress data has a median time sampling of $41 \mathrm{~s}$ and a standard deviation of 478 parts per million (ppm). The TS2 post-egress data has a median time sampling of $26 \mathrm{~s}$ and a standard deviation of $691 \mathrm{ppm}$. These noise levels are about $13 \%$ larger than the calculated noise due to photon-counting statistics, read noise, sky noise, and scintillation noise (using the approximate formulas of Reiger 1963 and Young 1967). The ratio of the observed TS1 noise to the observed TS2 noise is 0.69 , which is nearly equal to $\left(t_{\exp , 2} / t_{\exp , 1}\right)^{-1 / 2}=0.71$. The near-equality is evidence that the dominant noise source is a combination of photon noise and scintillation noise, both of which vary as $t_{\exp }^{-1 / 2}$. In the final version of TS3, which has a median time sampling of $41 \mathrm{~s}$, the pre-ingress data has an rms of $475 \mathrm{ppm}$ and the post-egress data has an rms of $488 \mathrm{ppm}$. These values are about $17 \%$ above the calculated noise level. In neither light curve did we detect signficant correlation between the noise and the pixel coordinates, FWHM, or shape parameters of the stellar images.

\section{Photometric Analysis}

We fitted a transit light curve model to the data, based on the analytic formulae of Mandel \& Agol (2002). The set of model parameters included the planet-to-star radius ratio $\left(R_{p} / R_{\star}\right)$, the ratio of the stellar radius to the orbital semimajor axis $\left(R_{\star} / a\right)$, the orbital inclination $(i)$, and the midtransit time $\left(T_{c}\right)$. We also fitted for three parameters $\left(\Delta m_{0}, k_{z}\right.$ and $k_{t}$ ) specifying a correction for systematic errors,

$$
\Delta m_{\mathrm{cor}}=\Delta m_{\mathrm{obs}}+\Delta m_{0}+k_{z} z+k_{t} t
$$

where $z$ is the airmass, $t$ is the time, $\Delta m_{\mathrm{obs}}$ is the observed magnitude difference between the target and comparison star, and $\Delta m_{\text {cor }}$ is the corrected magnitude difference that is compared to the idealized transit model. The $k_{z}$ term specifies the differential airmass extinction correction that was mentioned in $\oint 2$. The $k_{t}$ term was not strictly necessary to fit the data (it was found to be consistent with zero), but we included it in order to derive conservative error estimates for the transit times, as the parameter $k_{t}$ is covariant with the transit time. We allowed $\Delta m_{0}, k_{t}$, and $k_{z}$ to be specific to each time series, with the exception that TS1 and TS2 had a common value of $k_{z}$ because those data were obtained on the same night.

The final two model parameters were the coefficients $\left(u_{1}\right.$ and $\left.u_{2}\right)$ of a quadratic limbdarkening law,

$$
\frac{I_{\mu}}{I_{1}}=1-u_{1}(1-\mu)-u_{2}(1-\mu)^{2}
$$


where $\mu$ is the cosine of the angle between the line of sight and the normal to the stellar photosphere, and $I_{\mu}$ is the specific intensity. We allowed $u_{1}$ and $u_{2}$ to vary freely subject only to the conditions $u_{1}+u_{2}<1, u_{1}+u_{2}>0$, and $u_{1}>0$. It proved advantageous to perform the fit using the linear combinations

$$
v_{1}=u_{1}+2.33 u_{2}, \quad v_{2}=u_{1}-2.33 u_{2}
$$

because $v_{1}$ and $v_{2}$ have nearly uncorrelated errors (for further discussion, see Pál 2008).

We assumed the orbit to be circular because the RV data of Wilson et al. (2008) are consistent with a circular orbit, and because the expected timescale for orbital circularization at present,

$$
\tau_{c}=\frac{4}{63} Q_{p}\left(\frac{P}{2 \pi}\right)\left(\frac{M_{p}}{M_{\star}}\right)\left(\frac{a}{R_{p}}\right)^{5},
$$

(Rasio et al. 1996) is $0.3 \mathrm{Myr}\left(Q_{p} / 10^{5}\right)$ for WASP-4b, which is much shorter than the estimated main-sequence age of the star. In this expression, $Q_{p}$ is the the tidal dissipation parameter (see, e.g., Goldreich \& Soter 1966). This order-of-magnitude argument suggests that assuming a circular orbit is reasonable, although the value of $Q_{p}$ for irradiated giant planets is highly uncertain, and the expected timescale is highly approximate because it ignores the coupled evolution of the orbital distance and eccentricity (Jackson, Greenberg, \& Barnes 2008).

The fitting statistic was

$$
\chi_{F}^{2}=\sum_{i=1}^{N}\left[\frac{f_{i}(\mathrm{obs})-f_{i}(\text { calc })}{\sigma_{f, i}}\right]^{2},
$$

where $N$ is the number of flux measurements (photometric data points), $f_{i}(\mathrm{obs})$ is the $i$ th measurement, $f_{i}$ (calc) is the calculated flux given a particular choice of model parameters, and $\sigma_{f, i}$ is the uncertainty in the $i$ th measured flux. We determined appropriate values of $\sigma_{f, i}$ as follows. First, we multiplied the calculated errors in each time series by a constant chosen to give a minimum value of $\chi_{F}^{2} / N_{\text {dof }}=1$. The constants were $1.20,1.12$, and 1.21 for TS1, TS2, and TS3 respectively. Next, we assessed the time-correlated noise (also called "red noise") by examining the autocorrelation function, the power spectrum, and a plot of the Allan (1966) deviation of the residuals. We also used the method described by Winn et al. (2008), in which the ratio $\beta$ is computed between the standard deviation of timeaveraged residuals, and the standard deviation one would expect assuming white noise. For TS1 and TS2 we found no evidence for significant correlations. For TS3 we found structure in the autocorrelation function on a time scale of 15-20 min, the approximate ingress or egress duration, giving $\beta=1.52$. One naturally suspects that the correlated noise 
represents measurement error, although it is also possible that the noise is astrophysical, arising from starspots or other stellar inhomogeneities. In support of an astrophysical origin, we find no evidence for correlated noise $(\beta=1)$ when considering only the out-of-transit data. Nevertheless we cannot draw a firm conclusion; instead we attempt to account for the correlations by multiplying the error bars of TS 3 by an additional factor of $\beta=1.52$. Thus, for TS1, TS2, and TS3, the final values of $\sigma_{f, i}$ were equal to the calculated error bars multiplied by $1.20,1.12$, and $1.21 \times 1.52=1.84$, respectively. The error bars given in Table 1 are the final values of $\sigma_{f, i}$ that were used in the fitting process.

To determine the allowed ranges of each parameter, we used a Markov Chain Monte Carlo (MCMC) technique, with the Gibbs sampler and the Metropolis-Hastings algorithm, to estimate the a posteriori joint probability distribution of all the model parameters. This algorithm creates a sequence of points (a "chain") in parameter space by iterating a jump function, which in our case was the addition of a Gaussian random deviate to a randomlyselected single parameter. After this operation, if the new point has a lower $\chi_{F}^{2}$ than the previous point, the "jump" is executed: the new point is added to the chain. If not, then the jump is executed with probability $\exp \left(-\Delta \chi_{F}^{2} / 2\right)$. When the jump is not executed, the current point is repeated in the chain. The sizes of the random deviates are adjusted so that $\sim 40 \%$ of jumps are executed. After creating 10 chains of 500,000 links to check for mutual convergence, and trimming the first $20 \%$ of the links to eliminate artifacts of the initial conditions, the density of the chains' points in parameter space was taken to be the joint a posteriori probability distribution of the parameter values. Probability distributions for individual parameters are created by marginalizing over all other parameters.

The results are given in Table 2, which gives the median of each distribution, along with the $68.3 \%$ lower and upper confidence limits (defined by the $15.85 \%$ and $84.15 \%$ levels of the cumulative distribution). The entries designated $\mathrm{A}$ are those that follow directly from the photometric analysis. The entries designated B are those that are drawn from Gillon et al. (2008) and are repeated here for convenience. The entries designated C are based on a synthesis of our modeling results and theoretical models of stellar evolution, as discussed in $\S 4$. The entries designated $\mathrm{D}$ are the parameters of a refined transit ephemeris based on the two newly-measured transit times as well as some other available timing data (see $\S 5$ ).

As a consistency check we tried fitting the 2008 Aug 19 data and the 2008 Oct 09 data separately. We found that the results for the parameters $R_{p} / R_{\star}, R_{\star} / a, i, u_{1}$, and $u_{2}$ were all in agreement within $1 \sigma$, suggesting that our error estimates are reasonable. 


\section{Theoretical isochrone fitting}

The combination of transit photometry and the spectroscopic orbit (radial-velocity variation) of the host star do not uniquely determine the masses and radii of the planet and the star. There remain fitting degeneracies $M_{p} \propto M_{\star}^{2 / 3}$ and $R_{p} \propto R_{\star} \propto M_{\star}^{1 / 3}$ (see, e.g., Winn 2008). We broke these degeneracies by requiring consistency between the observed properties of the star, the stellar mean density $\rho_{\star}$ that can be derived from the photometric parameter $a / R_{\star}$ (Seager \& Mallen-Ornelas 2003, Sozzetti et al. 2007), and theoretical models of stellar evolution. The inputs were $T_{\text {eff }}=5500 \pm 100 \mathrm{~K}$ and $[\mathrm{Fe} / \mathrm{H}]=-0.03 \pm 0.09$ from Gillon et al. (2008), the stellar mean density $\rho_{\star}=1.694_{-0.037}^{+0.017} \mathrm{~g} \mathrm{~cm}^{-3}$ derived from the results for the $a / R_{\star}$ parameter, and the Yonsei-Yale $\left(\mathrm{Y}^{2}\right)$ stellar evolution models by Yi et al. (2001) and Demarque et al. (2004). We computed isochrones for the allowed range of metallicities, and for stellar ages ranging from 0.1 to 14 Gyr. For each stellar property (mass, radius, and age), we took a weighted average of the points on each isochrone, in which the weights were proportional to $\exp \left(-\chi_{\star}^{2} / 2\right)$ with

$$
\chi_{\star}^{2}=\left[\frac{\Delta[\mathrm{Fe} / \mathrm{H}]}{\sigma_{[\mathrm{Fe} / \mathrm{H}]}}\right]^{2}+\left[\frac{\Delta T_{\mathrm{eff}}}{\sigma_{T_{\mathrm{eff}}}}\right]^{2}+\left[\frac{\Delta \rho_{\star}}{\sigma_{\rho_{\star}}}\right]^{2} .
$$

Here, the $\Delta$ quantities denote the deviations between the observed and calculated values at each point. The asymmetric error bar in $\rho_{\star}$ was taken into account by using different values of $\sigma_{\rho_{\star}}$ depending on the sign of the deviation. The weights were further multiplied by a factor taking into account the number density of stars along each isochrone, assuming a Salpeter mass function. This procedure is essentially the same as that employed by Torres et al. (2008). The only difference is that we calculated the $68.3 \%$ uncertainties by assuming that the errors in $T_{\text {eff }}, \rho_{\star}$, and $[\mathrm{Fe} / \mathrm{H}]$ obey a Gaussian distribution, while Torres et al. (2008) took the distribution to be uniform within the quoted $1 \sigma$ limits.

Through this analysis, we found $M_{\star}=0.925 \pm 0.040 M_{\odot}$ and $R_{\star}=0.912 \pm 0.013 R_{\odot}$. The stellar age was poorly constrained, with a formally allowed range of $6.5 \pm 2.3$ Gyr and a nearly uniform distribution. The corresponding planetary mass and radius were obtained by merging the results for the stellar properties with the parameters determined in our photometric analysis, and with the stellar radial-velocity semiamplitude $K_{\star}=0.24 \pm 0.01 \mathrm{~km} \mathrm{~s}^{-1}$ measured by Wilson et al. (2008). The results are $M_{p}=1.237 \pm 0.064 M_{\mathrm{Jup}}$ and $R_{p}=1.365 \pm 0.021 R_{\mathrm{Jup}}$. Table 2 gives these results, along with the values for some other interesting parameters that can be derived from the preceding results. As a consistency check, we computed the implied stellar surface gravity and its uncertainty based on our analysis, finding $\log g=4.481 \pm 0.008$

where $g$ is in $\mathrm{cm} \mathrm{s}^{-2}$. This agrees with the spectroscopic determination of surface gravity, $\log g=4.3 \pm 0.2$, made by Wilson et al. (2008) based on an analysis of the widths of pressure-sensitive lines in the optical spectrum. 
It is important to keep in mind that the quoted error bars for the parameters designated $\mathrm{C}$ in Table 2 are based on the measurement errors only, and assume that the any systematic errors in the $\mathrm{Y}^{2}$ isochrones are negligible. As a limited test for the presence of such errors, Torres et al. (2008) tried analyzing transit data for 9 systems using isochrones computed by three different groups: the $\mathrm{Y}^{2}$ isochrones used here as well as those of Girardi et al. (2000) and Baraffe et al. (1998). They found the differences to be smaller than the error bars, especially for stars similar to the Sun such as WASP-4. Similar results were found by Southworth (2009). Nevertheless, the true systematic errors in the $\mathrm{Y}^{2}$ isochrones are not known, and we have not attempted to quantify them here, although it seems plausible that the masses and radii are subject to an additional error of a few percent.

\section{Transit Times and a Refined Ephemeris}

Precise measurements of transit times are important because the gravitational perturbations from other bodies in the system (such as a satellite or additional planet) could produce detectable variations in the orbital period. Based on the MCMC analysis described in the previous section, the uncertainties in the two transit times are $4.6 \mathrm{~s}$ and $4.9 \mathrm{~s}$, making them among the most precise such measurements that have been achieved. This is a consequence of good photometric precision and fine time sampling, along with the relative insignificance of time-correlated noise and the large transit depth.

As a check on the error bars, we also estimated the midtransit time and its error using the residual permutation (RP) method, a type of bootstrap analysis that attempts to account for time-correlated errors. Fake data sets are created by subtracting the best-fitting model from the data, then adding the residuals back to the model after performing a cyclic permutation of their time indices. For each fake data set, $\chi_{F}^{2}$ is minimized as a function of $T_{c}, \Delta m_{0}, k_{z}$ and $k_{t}$ (the other parameters being held constant, as they are nearly uncorrelated with $T_{c}$ ). The distribution of the results for $T_{c}$ is taken to be the a posteriori probability distribution for $T_{c}$. For the 2008 Aug 19 data, the RP-based error bar was only $4 \%$ larger than the MCMC-based error bar; the results were nearly indistinguishable. For the 2008 Oct 09 data, the RP-based error was $25 \%$ larger than the MCMC-based error. To be conservative, we report the larger RP-based errors in Table 1 and used those larger error bars in recomputing the ephemeris (see below).

As a further check on the analysis, we allowed TS1 and TS2 to have independent values of $T_{c}, \Delta m_{0}, k_{z}$, and $k_{t}$ during the fitting process. In other words we fitted all the data but did not require that TS1 and TS2 agree on the transit time. The result was that the difference between $T_{c}(\mathrm{TS} 1)$ and $T_{c}$ (TS2) was $2.3 \pm 13.4 \mathrm{~s}$. We also tried a similar experiment 
with the 2008 Aug 19 data, splitting it into two equal parts that were fitted jointly with TS1 and TS2 (which in this case were required to agree on the midtransit time so as to provide a constraint on the transit duration). The result in this case was $\Delta T_{c}=4.6 \pm 11.9 \mathrm{~s}$. The mutual consistency of the results suggests that our error bars are reasonable.

The transits of 2008 Aug 19 and 2008 Oct 09 were separated by 38 orbital periods. By calculating $\Delta T_{c} / 38$ we derive an independent estimate of the orbital period, $P=1.3382369 \pm$ 0.0000024 days. The most precise determination previously reported was $P=1.3382324 \pm$ 0.0000029 days, by Gillon et al. (2008), based on 2 years of data. Our period has comparable precision, though it is based on only 2 transits separated by 50 days. The difference between the two independent period determinations is $0.39 \pm 0.32 \mathrm{~s}$.

We fitted a linear function of epoch to the two newly-measured midtransit times along with the 5 transit times given in Table 2 of Gillon et al. (2008). The fit had $\chi^{2}=7.8$ with 5 degrees of freedom. The chance of finding a value of $\chi^{2}$ this large by chance is $17 \%$, using the quoted $1 \sigma$ error bars and assuming the errors obey a Gaussian distribution. We deem this an acceptable fit, and conclude that the available data do not provide compelling evidence for any departures from a constant period. The refined transit ephemeris is $T_{c}(E)=T_{c}(0)+E P$, with

$$
\begin{aligned}
T_{c}(0) & =2,454,697.797562 \pm 0.000043[\mathrm{BJD}], \\
P & =1.33823214 \pm 0.00000071 .
\end{aligned}
$$

Fig. 2 shows a plot of the differences between the observed and calculated transit times.

\section{Discussion}

Our results for the planetary and stellar properties are in accord with the previous analyses by Wilson et al. (2008) and Gillon et al. (2008). In general our error bars are comparable in size or smaller than those of Gillon et al. (2008), who observed a transit with one of the Very Large Telescopes. This consistency is reassuring, especially since our error estimates are more conservative in some respects. We have accounted for uncertainty in the limb-darkening coefficients, as well as the slopes of systematic trends with time and airmass. Previous investigators assumed that these parameters were known exactly, leading to underestimated errors in any covariant parameters. Southworth (2008) demonstrated this effect for the limb-darkening coefficients in particular 1 In addition, Gillon et al. (2008)

\footnotetext{
${ }^{1}$ Our results for the limb-darkening coefficients are $u_{1}=0.311 \pm 0.041$ and $u_{2}=0.227 \pm 0.089$. These are not too far from the tabulated values 0.265 and 0.303 given by Claret (2004) for a star with the observed
} 
Table 1. Relative Photometry of WASP-4

\begin{tabular}{ccc}
\hline \hline Barycentric Julian Date & Relative flux & Uncertainty \\
\hline 2454697.708386 & 1.00005 & 0.00050 \\
2454697.708861 & 1.00018 & 0.00050 \\
2454697.709337 & 1.00003 & 0.00050 \\
2454697.709810 & 0.99975 & 0.00050 \\
2454697.710285 & 0.99930 & 0.00050 \\
2454697.710759 & 0.99996 & 0.00049 \\
2454697.711234 & 0.99917 & 0.00050 \\
2454697.711708 & 1.00067 & 0.00050 \\
2454697.712185 & 1.00039 & 0.00050 \\
2454697.712657 & 1.00066 & 0.00049 \\
2454697.713132 & 1.00062 & 0.00050 \\
2454697.713606 & 0.99982 & 0.00050 \\
2454697.714082 & 0.99976 & 0.00049 \\
\hline
\end{tabular}

Note. - The time stamps in Column 1 represent the Barycentric Julian Date at the time of midexposure. The uncertainty in Column 3 is the quantity $\sigma_{f, i}$ discussed in $\S 3$. We intend for this Table to appear in entirety in the electronic version of the journal. An excerpt is shown here to illustrate its format. The data are also available from the authors upon request.

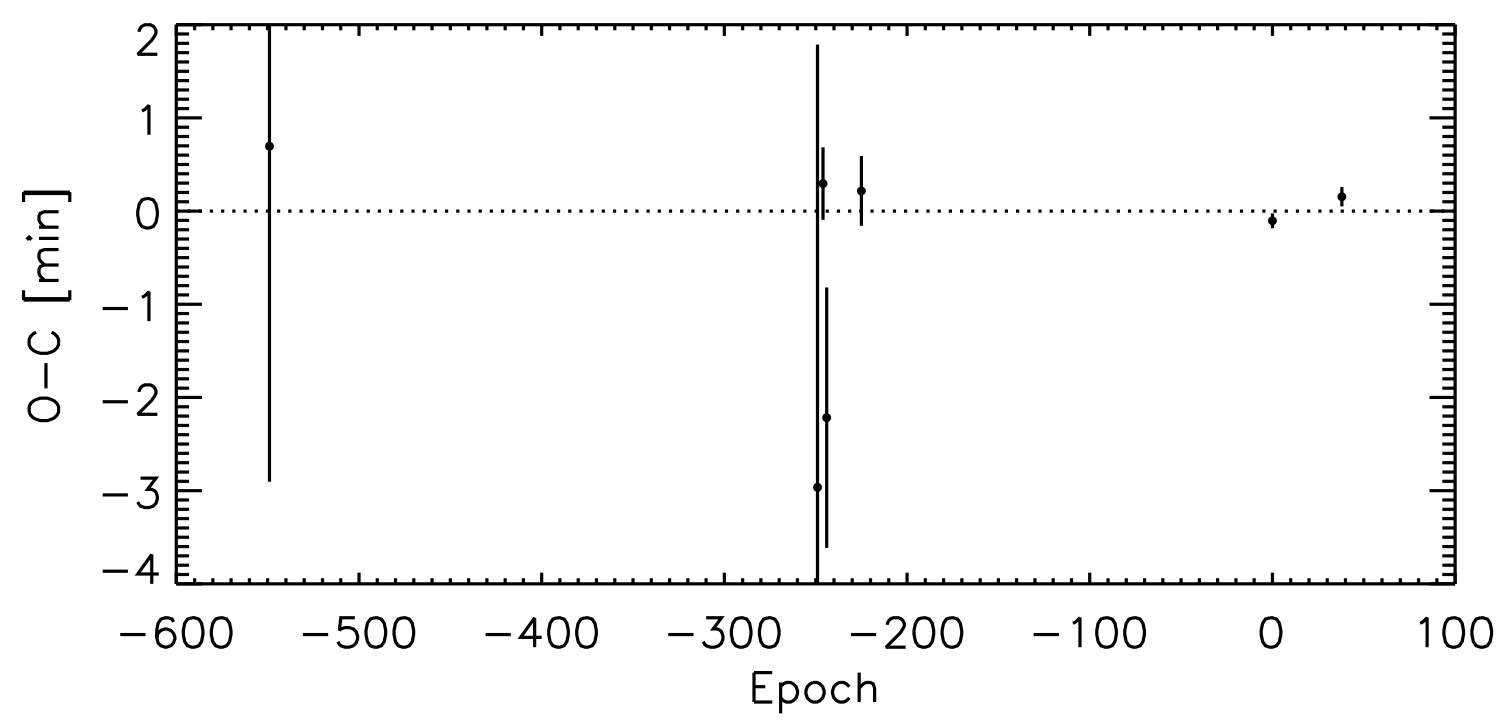

Fig. 2.- Transit timing residuals for WASP-4b. The calculated times (using the ephemeris given in Eq. (7) have been subtracted from the observed times. 
Table 2. System Parameters of WASP-4b

\begin{tabular}{|c|c|c|c|}
\hline Parameter & Value & $68.3 \%$ Conf. Limits & Comment \\
\hline \multicolumn{4}{|l|}{ Transit ephemeris: } \\
\hline Reference epoch [BJD] & 2454697.797562 & \pm 0.000043 & $\mathrm{D}$ \\
\hline Orbital period [days] & 1.33823214 & \pm 0.00000071 & $\mathrm{D}$ \\
\hline \multicolumn{4}{|l|}{ Transit parameters: } \\
\hline Midtransit time on 2008 Aug 19 [BJD] & 2454697.797489 & \pm 0.000055 & A \\
\hline Midtransit time on 2008 Oct 09 [BJD] & 2454748.650490 & \pm 0.000072 & A \\
\hline Planet-to-star radius ratio, $R_{p} / R_{\star}$ & 0.15375 & $-0.00055,+0.00077$ & A \\
\hline Orbital inclination, $i$ [deg] & 88.56 & $-0.46,+0.98$ & A \\
\hline Scaled semimajor axis, $a / R_{\star}$ & 5.473 & $-0.051,+0.015$ & A \\
\hline Transit impact parameter, $b=a \cos i / R_{\star}$ & 0.143 & $-0.098,+0.038$ & A \\
\hline Transit duration $[\mathrm{hr}]$ & 2.1660 & $-0.0062,+0.0054$ & $\mathrm{~A}$ \\
\hline Transit ingress or egress duration $[\mathrm{hr}]$ & 0.2913 & $-0.0002,+0.01006$ & A \\
\hline Linear limb-darkening coefficient, $u_{1}$ & 0.311 & \pm 0.041 & $\mathrm{~A}$ \\
\hline Quadratic limb-darkening coefficient, $u_{2}$ & 0.227 & \pm 0.089 & $\mathrm{~A}$ \\
\hline Semimajor axis $[\mathrm{AU}]$ & 0.02340 & \pm 0.00060 & $\mathrm{C}$ \\
\hline Planet-to-star mass ratio, $M_{p} / M_{\star}$ & 0.00127 & \pm 0.00012 & $\mathrm{C}$ \\
\hline \multicolumn{4}{|l|}{ Stellar parameters: } \\
\hline Mass, $M_{\star}\left[\mathrm{M}_{\odot}\right]$ & 0.925 & \pm 0.040 & $\mathrm{C}$ \\
\hline Radius, $R_{\star}\left[\mathrm{R}_{\odot}\right]$ & 0.912 & \pm 0.013 & $\mathrm{C}$ \\
\hline Surface gravity, $\log g_{\star}[\mathrm{cgs}]$ & 4.4813 & \pm 0.0080 & $\mathrm{C}$ \\
\hline Mean density, $\rho_{\star}\left[\mathrm{g} \mathrm{cm}^{-3}\right]$ & 1.728 & $-0.047,+0.016$ & A \\
\hline Effective temperature, $T_{\text {eff }}[\mathrm{K}]$ & 5500 & \pm 100 & $\mathrm{~B}$ \\
\hline Metallicity, $[\mathrm{Fe} / \mathrm{H}]$ & -0.03 & \pm 0.09 & $\mathrm{~B}$ \\
\hline Projected rotation rate, $v \sin i_{\star}\left[\mathrm{km} \mathrm{s}^{-1}\right]$ & 2.0 & \pm 1.0 & $\mathrm{~B}$ \\
\hline Luminosity $\left[\mathrm{L}_{\odot}\right]$ & 0.682 & \pm 0.065 & $\mathrm{C}$ \\
\hline Absolute $V$ magnitude & 5.30 & \pm 0.13 & $\mathrm{C}$ \\
\hline Age [Gyr] & 6.5 & \pm 2.3 & $\mathrm{C}$ \\
\hline \multicolumn{4}{|l|}{ Planetary parameters: } \\
\hline$M_{p}\left[\mathrm{M}_{\mathrm{Jup}}\right]$ & 1.237 & \pm 0.064 & $\mathrm{C}$ \\
\hline$R_{p}\left[\mathrm{R}_{\mathrm{Jup}}\right]$ & 1.365 & \pm 0.021 & $\mathrm{C}$ \\
\hline Surface gravity, $g_{p}\left[\mathrm{~m} \mathrm{~s}^{-2}\right]$ & 16.41 & \pm 0.75 & $\mathrm{~A}$ \\
\hline Mean density, $\rho_{p}\left[\mathrm{~g} \mathrm{~cm}^{-3}\right]$ & 0.604 & \pm 0.042 & $\mathrm{C}$ \\
\hline
\end{tabular}

Note. - (A) Based on the analysis of the new light curves (see § 3). (B) From Gillon et al. (2008). (C) Functions of group A and B parameters, supplemented by theoretical $\mathrm{Y}^{2}$ isochrones (see $\S 4$ ). For (C) parameters, the error bars are based only on the measurement errors and do not account for any possible error in the $\mathrm{Y}^{2}$ isochrones. (D) Based on the two newly-measured transit times, as well as all entries in Table 2 of Gillon et al. (2008). 
found a much stronger influence of correlated noise on the results for the midtransit time. In the language of $\S 3$, their RP-based error bar was 300\%-400\% larger than the MCMCbased error bar, as compared to $4-25 \%$ in our case. We also question the applicability of the "small-planet" approximation used by Gillon et al. (2008). With our data, using the small-planet approximation would have led to an erroneous $2.3 \sigma$ shift in $R_{p} / R_{\star}$.

For the key parameter $R_{p}$, Wilson et al. (2008) found $1.416_{-0.043}^{+0.068} R_{\text {Jup }}$, Gillon et al. (2008) found $1.304_{-0.042}^{+0.054} R_{\mathrm{Jup}}$, and we find $1.365 \pm 0.021 R_{\mathrm{Jup}}$. Here it is important to reiterate that the estimate of $R_{p}$ relied on the $\mathrm{Y}^{2}$ isochrones, which probably contribute an additional systematic error of a few percent. Despite this, it seems clear that WASP-4b is a member of the class of "bloated" planets, by which we mean planets that are larger than predicted according to theoretical models of solar-composition gas giant planets, even after accounting for the intense irradiation from the parent star.

Bodenheimer et al. (2003) predicted the radii of giant planets as a function of the age, mass, and equilibrium temperature, defined as

$$
T_{\text {eq }}=\left[\frac{(1-A) L_{\star}}{16 \pi \sigma a^{2}}\right]^{1 / 4}=(1677 \mathrm{~K})(1-A)^{1 / 4}
$$

where $A$ is the Bond albedo, $L_{\star}$ is the stellar luminosity, $\sigma$ is the Stefan-Boltzmann constant, and $a$ is the semimajor axis. In the latter equality we have evaluated $T_{\text {eq }}$ for WASP-4b using the results given in Table 2. As long as the albedo is not very close to unity, Bodenheimer et al. (2003) predict a planetary radius of $1.13 R_{\text {Jup }}$ for a solar composition at $4.5 \mathrm{Gyr}$. This is smaller than the observed radius by $11 \sigma$.

Fortney et al. (2007) presented theoretical models parameterized by mass, age, and an effective orbital distance, defined as the distance from the Sun where a hypothetical planet would receive the same flux as the actual planet,

$$
a_{\oplus}=a\left(\frac{L_{\star}}{L_{\odot}}\right)^{-1 / 2}=0.0281 \mathrm{AU}
$$

where again we have evaluated the expression as appropriate for WASP-4b. For a solar composition at $4.5 \mathrm{Gyr}$, Fortney et al. (2007) predict $R_{p}=1.16 R_{\text {Jup }}$, about $10 \sigma$ smaller than the observed value. Neither of these sets of models takes into account the "transit

effective temperature and surface gravity. However, with data this precise, one should not fix the limbdarkening coefficients at the tabulated values. The tables have unquantified errors and these errors are strongly correlated with the other photometric parameters. For example, for HD 209458 the tabulated values can be ruled out with $>99.9 \%$ precision (Southworth 2008). 
radius effect" of Burrows et al. (2003) - the underprediction of transit radii by modelers who use an inappropriately high pressure to define the radius of their models - but this effect is expected to be only a few percent according to Fortney et al. (2007).

One might suppose that the system is young, and has therefore not had as much time to contract from its presumably hot and distended initial state. For an age of $300 \mathrm{Myr}$, Fortney et al. (2007) predict a radius of $1.30 R_{\text {Jup }}$, only $3 \sigma$ smaller than the observed value (and perhaps consistent, given the systematic errors in the isochrones and the transit radius effect). However, such a young age is disfavored by our isochrone analysis, and Wilson et al. (2008) have also argued that the star is older than about 2 Gyr based on a nondetection of Li I $(6708 \AA)$ in the spectrum.

Another possible resolution is to invoke an extra source of internal heat (of unspecified origin) within the planet. Liu et al. (2008) have provided useful fitting formulas to gauge how much extra power is required, for a solar-composition planet of a given mass and effective orbital distance. They are parameterized by the variable $x$, defined as the ratio of the extra power to the incident power from the host star's radiation. By interpolating Table 3 of Liu et al. (2008) we find that to match the observed radius of WASP-4b, one needs an "anomalous" heating rate of $\sim 0.15 \%$ of the incident power from the host star, or $\sim 8 \times 10^{26} \mathrm{erg} \mathrm{s}^{-1}$. According to the same tables, the equilibrium radius is achieved in $\sim 30 \mathrm{Myr}$.

Of course the preceding calculation does not solve the problem of the bloated planet. It merely quantifies the power requirement for the unknown heat source. The calculations of Liu et al. (2008) were in the context of tidal heating due to orbital circularization. According to their Eq. (A3), an orbital eccentricity of $0.002\left(Q_{p} / 10^{5}\right)$ would suffice to produce enough power to inflate the planet to the requisite degree. Hence for $Q_{p}$ between $10^{5}$ and $10^{6}$, the required eccentricity for this hypothesis is about 0.002 to 0.02 . This is small enough to be compatible with the RV data, but larger than expected based on the estimated stellar age and the order-of-magnitude timescale for tidal circularization (see $\S 3$ ), unless there is a third body whose gravitational influence is preventing circularization. A very precise measurement of the time of occultation of WASP-4b by its host star might allow the orbital eccentricity (or more precisely $e \cos \omega$, where $\omega$ is the argument of pericenter) to be measured well enough to test this notion. Of course, many other ways to explain the radii of the bloated planets have been given in the literature (Guillot \& Showman 2002, Showman \& Guillot 2002, Bodenheimer et al. 2003, Burrows et al. 2007, Chabrier \& Baraffe 2007). This issue remains unresolved for the particular case of WASP-4b and for the entire ensemble.

In addition to refining the estimates of planetary parameters, and seeking additional bodies through transit timing, an important application of precise differential photometry is checking for transits of planets that have been detected by the Doppler technique but for 
which the orbital inclination is unknown. Given the progress of the Doppler surveys in finding lower-mass planets, it is also a likely route to the discovery of the first examples of "superEarth" planets that transit bright stars, and that are therefore valuable for understanding the sizes, orbits, and atmospheres of terrestrial planets. It is generally thought that detection of transits of terrestrial-mass planets must be done from space, to eliminate the contaminating effects of Earth's atmosphere and to observe for long intervals without interruptions due to bad weather and the day/night cycle. These are clear advantages but they must be weighed against the high cost of space missions.

It is interesting to contemplate how small a planet we would have been able to detect, given data of the quality presented here. Our light curve has a signal-to-noise ratio of approximately $\sqrt{\Gamma T} \delta / \sigma \approx 920$, using the notation of Carter et al. (2008) in which $\Gamma T$ is the number of data points per transit duration, $\delta$ is the transit depth, and $\sigma$ the photometric precision. Statistically one might expect that we would have achieved a $5 \sigma$ detection of a planet that has an area 920/5 = 184 times smaller than WASP-4b, i.e., a planet with $R_{p}=1.1 R_{\oplus}$. However, it is not clear whether a statistically unassailable $5 \sigma$ detection would really be credible, given the possible presence of systematic trends, correlated errors, uncertainty in predicted transit times, and general caution.

To judge the believability of a super-Earth detection, we added to the residuals of Fig. 2 a model light curve of a transiting super-Earth. All of the parameters of the model were the same as the actual WASP-4b parameters except the planetary radius, which was reduced to $1.75 R_{\oplus}$, the approximate upper limit that is predicted for terrestrial planets. The result, shown in Fig. 3, gives a visual impression of what one might realistically expect, based on two nights of observations of a 12th magnitude star with a large telescope.

We fitted this simulated data in nearly the same way that we fitted the WASP-4 data. The only difference is that we fixed the values of the limb-darkening parameters and the stellar radius, as befits a detection rather than a characterization experiment. We also assumed the orbital period was known to within $10 \%(3.2 \mathrm{hr})$. The free parameters were $\left(R_{p} / R_{\star}\right)$; $i$; the two mid-transit times; and the parameters describing systematic trends, $\Delta m_{0}, k_{z}$ and $k_{t}$, for each time series. The results were that $\left(R_{p} / R_{\star}\right)$ was consistent with the input value and had an error of $14 \%$, dominated by the correlation with the poorlyconstrained orbital inclination. The transit times were recovered within $5 \mathrm{~min}$. The detection is visually apparent in the time-binned light curve shown in the lower panel of Fig. 3. One would certainly prefer a longer stretch of out-of-transit data, especially if the orbital period has a large uncertainty. Nevertheless the impression given is that the detection of transits of super-Earths around Sun-like stars is within reach of ground-based observations. 

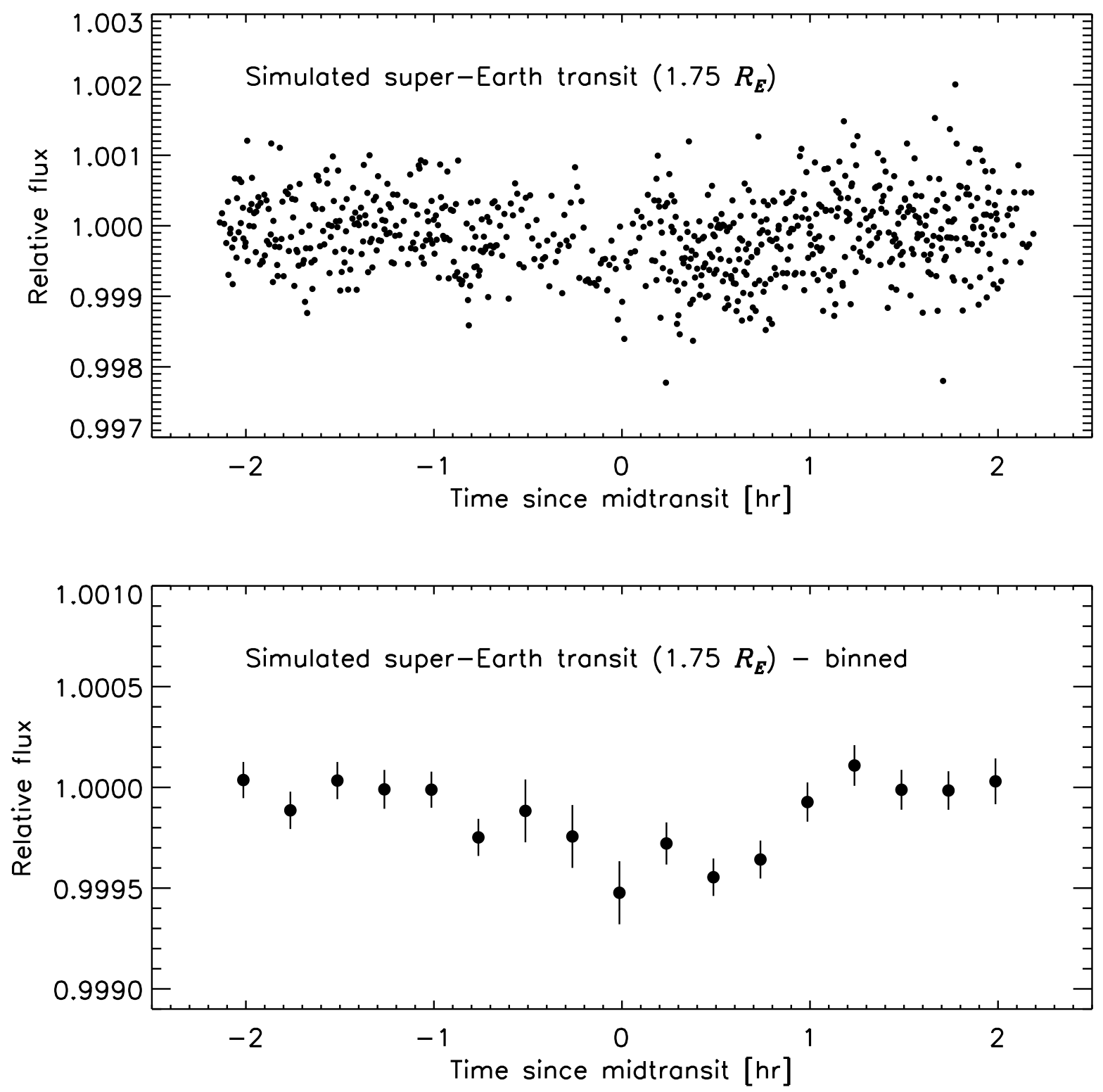

Fig. 3.- Simulated photometric observations of a transiting super-Earth around a 12th magnitude G7V star, based on our WASP-4 data. The residuals shown in Fig. 1 were added to an idealized transit model in which $R_{p}=1.75 R_{\oplus}$ and all other parameters were the same as in the WASP-4 model. The bottom panel shows a time-binned version of the simulated data. 
We thank Paul Schechter and Adam Burgasser for observing on our behalf on several occasions, for this project and related projects, and for helpful discussions on observing techniques. Partial support for this work came from NASA Origins grants NNX09AB33G (to M.J.H.) and NNG04LG89G (to G.T.).

\section{REFERENCES}

Agol, E., Steffen, J., Sari, R., \& Clarkson, W. 2005, MNRAS, 359, 567

Allan, D. W. 1966, Proc. IEEE, 54, 221

Baraffe, I., Chabrier, G., Allard, F., \& Hauschildt, P. H. 1998, A\&A, 337, 403

Bodenheimer, P., Laughlin, G., \& Lin, D. N. C. 2003, ApJ, 592, 555

Burrows, A., Sudarsky, D., \& Hubbard, W. B. 2003, ApJ, 594, 545

Burrows, A., Hubeny, I., Budaj, J., \& Hubbard, W. B. 2007, ApJ, 661, 502

Carter, J. A., Yee, J. C., Eastman, J., Gaudi, B. S., \& Winn, J. N. 2008, ApJ, 689, 499

Chabrier, G., \& Baraffe, I. 2007, ApJ, 661, L81

Claret, A. 2004, A\&A, 428, 1001

Demarque, P., Woo, J.-H., Kim, Y.-C., \& Yi, S. K. 2004, ApJS, 155, 667

Fortney, J. J., Marley, M. S., \& Barnes, J. W. 2007, ApJ, 659, 1661

Gillon, M., et al. 2008, arXiv:0812.1998

Girardi, L., Bressan, A., Bertelli, G., \& Chiosi, C. 2000, A\&AS, 141, 371

Goldreich, P., \& Soter, S. 1966, Icarus, 5, 375

Guillot, T., \& Showman, A. P. 2002, A\&A, 385, 156

Guillot, T. 2008, Physica Scripta Volume T, 130, 014023

Holman, M. J., \& Murray, N. W. 2005, Science, 307, 1288

Holman, M. J., et al. 2006, ApJ, 652, 1715

Jackson, B., Greenberg, R., \& Barnes, R. 2008, ApJ, 678, 1396 
Liu, X., Burrows, A., \& Ibgui, L. 2008, ArXiv e-prints, 805, arXiv:0805.1733

Mandel, K., \& Agol, E. 2002, ApJ, 580, L171

Pál, A. 2008, MNRAS, 1043

Rasio, F. A., Tout, C. A., Lubow, S. H., \& Livio, M. 1996, ApJ, 470, 1187

Reiger, S. H. 1963, AJ, 68, 395

Sasselov, D. D. 2003, ApJ, 596, 1327

Seager, S., \& Mallén-Ornelas, G. 2003, ApJ, 585, 1038

Showman, A. P., \& Guillot, T. 2002, A\&A, 385, 166

Southworth, J. 2008, MNRAS, 386, 1644

Southworth, J. 2009, arXiv:0811.3277

Sozzetti, A., Torres, G., Charbonneau, D., Latham, D. W., Holman, M. J., Winn, J. N., Laird, J. B., \& O’Donovan, F. T. 2007, ApJ, 664, 1190

Torres, G., Winn, J. N., \& Holman, M. J. 2008, ApJ, 677, 1324

Wilson, D. M., et al. 2008, ApJ, 675, L113

Winn, J. N., et al. 2007, AJ, 133, 1828

Winn, J. N. 2008, ArXiv e-prints, 807, arXiv:0807.4929

Winn, J. N., et al. 2008, ApJ, 683, 1076

Yi, S., Demarque, P., Kim, Y.-C., Lee, Y.-W., Ree, C. H., Lejeune, T., \& Barnes, S. 2001, ApJS, 136, 417

Young, A. T. 1967, AJ, 72, 747 